\title{
ELECTRICAL ACTUATION AT NANOSCALE: CONTROLLED ORIENTATION OF PROTEINS DURING IMMOBILIZATION
}

\author{
Sam Emaminejad ${ }^{1,2,3^{*}}$, Mehdi Javanmard ${ }^{1,2,3^{*}}$, Shuai Chang ${ }^{l}$, Chaitanya Gupta ${ }^{l}$, \\ Ronald W. Davis ${ }^{2,3}$, and Roger T. Howe ${ }^{l}$ \\ ${ }^{1}$ Electrical Engineering Department, Stanford University, CA, USA \\ ${ }^{2}$ School of Medicine, Stanford University, CA, USA \\ ${ }^{3}$ Stanford Genome Technology Center, Stanford University, Palo Alto, CA, USA \\ * Equal Contribution
}

\begin{abstract}
Here, we demonstrate the ability to control protein orientation using electrical field. We used atomic force microscopy (AFM) to verify that the application of electric field results in modulation of the orientation of antibodies (IgG molecules) and also enhancement of immobilization. We also demonstrate the applicability of this technique to improve the performance of fluorescent affinity biosensors.
\end{abstract}

\section{INTRODUCTION}

We previously [1] demonstrated a three stage microfluidic sample preparation platform for filtering cells and highly abundant proteins (using enhanced dielectrophoresis force $[2,3]$ ) and presented preliminary results for orienting target proteins for probe-free biosensing platforms. Here, we show significant improvement in the ability to control protein orientation using electrical field. We used atomic force microscopy (AFM) to verify that the application of electric field results in modulation of the orientation of antibodies ( $\mathrm{IgG}$ molecules) and also enhancement of immobilization. We also demonstrate the applicability of this technique to improve the performance of fluorescent affinity biosensors. Previously Talasaz et al. [4] developed a simulation procedure predicting protein orientation related to its immobilization affected by the electric field at a solidstate support. Here, we exploit the dipole property of $\mathrm{IgG}$ and its ability to be oriented with field (Figure 1A,B).
A)

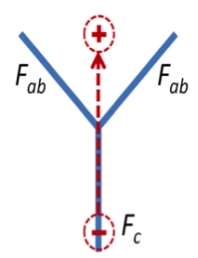

B)

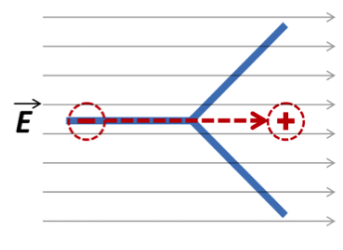

Figure 1: A) Antibodies ( $\mathrm{IgG}$ ) have an $\mathrm{Fc}_{\mathrm{C}}$ region and an Fab region where the isoelectric point of the Fab region is higher compared to the Fab resulting in dipole behavior. B) The antibody reorients itself in the field direction.

\section{METHODS}

We study the orientation of antibodies in rectangular microchannels in order to ensure uniform electric field across the channel (fabricated in PDMS $200 \mu \mathrm{m}$ wide, $50 \mu \mathrm{m}$ high, and $1 \mathrm{~cm}$ long). We apply electric field across the channel while physically adsorbing antibodies $(0.02 \mathrm{mg} / \mathrm{ml})$ to the base of the channel (Figure 2). We inserted $\mathrm{Ag}-\mathrm{AgCl}$ electrodes at the inlet and outlet ports of the microchannel. We excited the electrodes with DC voltages up to $8 \mathrm{~V}$. We independently optically verified that the voltages were not high enough to induce either electroosmotic flow or electrophoretic flow. We then removed the channel and scanned the surface with AFM.

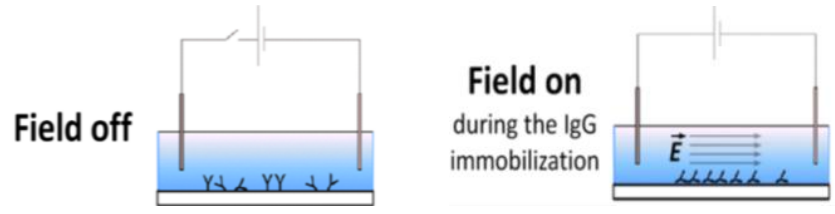

Figure 2: Schematic of AFM assay setup. Antibodies oriented with field during the immobilization step, then imaged with AFM.

\section{RESULTS AND DISCUSSION}

Our AFM images illustrated that application of electric field results in uniform orientation and lining up of the antibodies during the immobilization step (Figure 3), compared to the case where electric field is off and molecules become randomly oriented on the surface.

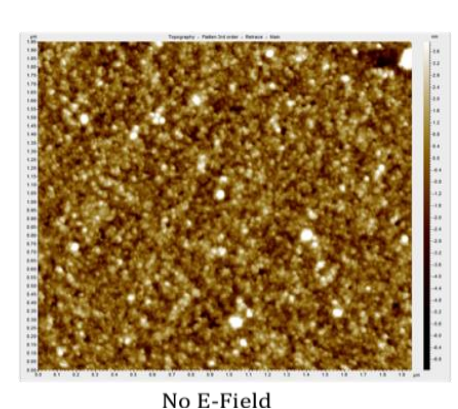

A)

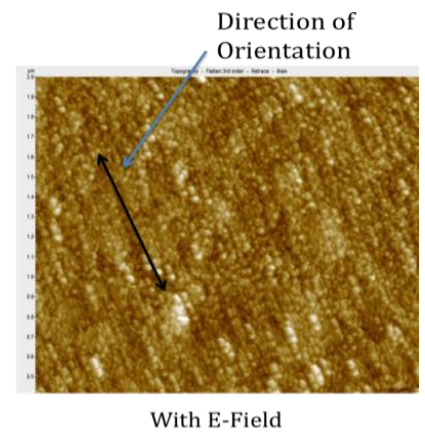

B)
Figure 3: Comparison of antibody coated surface when field is off during immobilization to when field is on. A) Two dimensional AFM image for when field is off. B) Two dimensional AFM image for when field is on. Results indicated that field results in antibodies orienting in a uniform direction.

We diluted the IgG sample even further (1000X) in order to be able to image single antibodies. Results clearly showed that IgG molecules were uniformly oriented in a single direction (Figure 4) as predicted by our hypothesis (Figures 1 and 2).

We also apply this novel method in controlling the orientation of proteins to improve biosensor sensitivity. In the case of affinity-based sensors, the probe protein has specific sites or epitopes where binding occurs. Probe antibody orientation during immobilization onto the surface affects efficiency of target analyte capture. As an example, we studied the interaction of fluorescently tagged (FITC) anti-IgG with surface immobilized IgG (0.02 $\mathrm{mg} / \mathrm{ml}$ ), where only the immobilization process is controlled by electric field (Figure 5). 


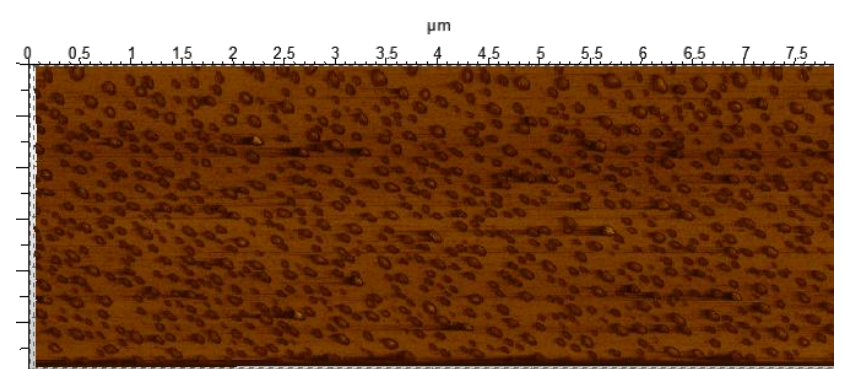

A)

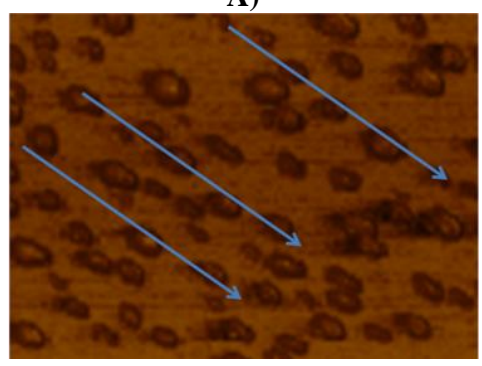

B)

Figure 4: A) IgG concentration diluted by $1000 X$ so that single antibodies can be visualized with AFM. Antibodies are all oriented in the same direction. B) A zoomed in view of the original AFM image shown in part $A$ with the arrows showing the orientation of molecules.

All positive and negative control experiments were performed in parallel. Our negative control was the case where no electrodes were inserted in the channel during $\mathrm{IgG}$ immobilization. The representative captured snapshots from our experiments illustrate enhancement in the binding of anti-IgG and $\operatorname{IgG}$ as a result of applying lateral electric field during $\operatorname{IgG}$ immobilization (Figure 6A). The collective results for the fluorescence assay are presented in Figure 6B, which shows the improvement in signal intensity as we increased applied voltage. Our study demonstrates that the use of electric field can result in up to $40 \mathrm{X}$ enhancement in signal to noise ratio compared to normal physical adsorption. The improvement in sensitivity results both from favorable orientation of the antibody and also increase in the density of antibodies immobilized on the surface.

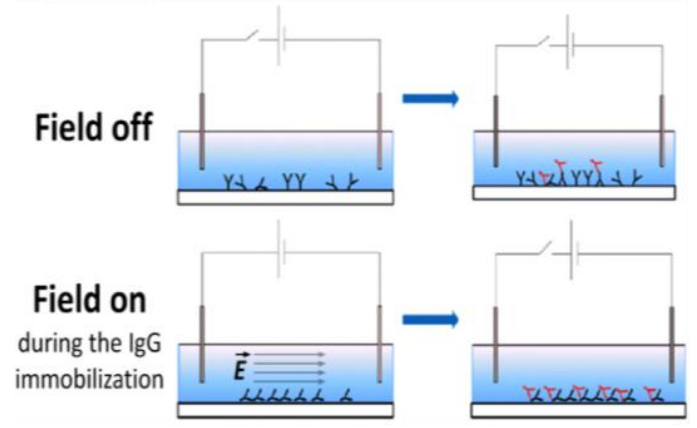

Figure 5: Fluorescent assay experimental setup. Field is modulated during the $\operatorname{Ig} G$ immobilization. For the case where electric field is off IgG molecules are randomly oriented. For the case where field is on, IgG molecules are oriented on their sides so that the $F_{c}$ region is exposed allowing an increase in binding of anti-goat IgG molecules.

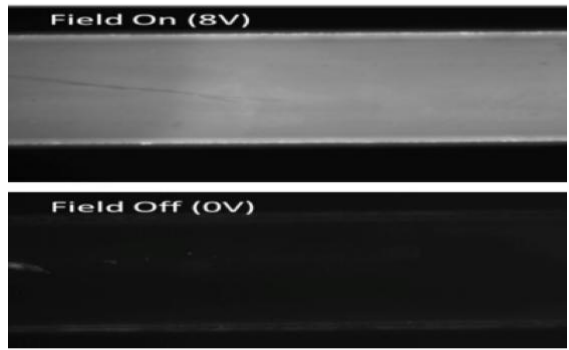

A)

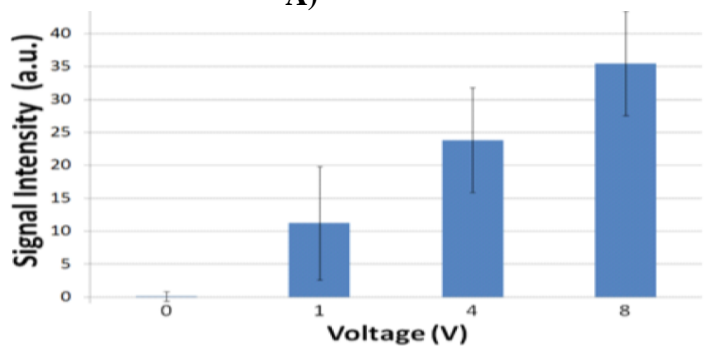

B)

Figure 6: A) The representative snapshots from our experiments illustrating enhancement in the binding of anti-IgG and $\operatorname{Ig} G$ as a result of applying lateral electric field during IgG immobilization. B) Corresponding relationship of the fluorescent intensity verses the voltage applied, demonstrating the improvement in signal intensity as we increased applied voltage

\section{ACKNOWLEDGEMENTS}

This work was supported by the Defense Advanced Research Projects Agency grant DARPA BAA-11-07. Fabrication of the devices was performed in the Stanford Nanofabrication Facility.

\section{REFERENCES}

[1] M. Javanmmard, S. Emaminejad, C. Gupta, J. Provine, R.W. Davis, and R.T. Howe, "Depletion of Cells and Abundant Proteins from Biological Samples by Enhanced Dielectrophoresis", Sensors and Actuators B: Chemical, vol. 193, pp. 918-924 (2013).

[2] S. Emaminejad, M. Javanmmard, R.W. Dutton, and R.W. Davis, "Use of Negative Dielectrophoresis for Selective Elution of Protein-Bound Particles", Analytical Chemistry, 84 (3), pp 1432-1438 (2012).

[3] S. Emaminejad, M. Javanmmard, R.W. Dutton, and R.W. Davis, "Smart Surface for Elution of Protein-Protein Bound Particles: Nanonewton Dielectrophoretic Forces Using Atomic Layer Deposited Oxides", Analytical Chemistry, 84 (24), pp 10793-10801 (2012).

[4] A. H. Talasaz, M. Nemat-Gorgani, Y. Liu, P. Stahl, R. W. Dutton, M. Ronaghi, and R. W. Davis, "Prediction of protein orientation upon immobilization on biological and nonbiological surfaces", Proc. Natl. Acad. Sci. (103) 14773 (2006).

\section{CONTACT}

*S. Emaminejad, same@stanford.edu 